\title{
Respostas Fisiológicas Associadas à Termotolerância em Pintos de Corte de Duas Linhagens por Exposição a Altas Temperaturas
}

\section{Nelson José Laurino Dionello1,4, Marcos Macari², Jesus Aparecido Ferro ${ }^{3}$, Fernando Rutz ${ }^{4}$, Maria Inês Tiraboschi Ferro ${ }^{3}$, Luiz Roberto Furlan ${ }^{5}$}

\begin{abstract}
RESUMO - Com o objetivo de se avaliar o comportamento fisiológico das aves, bem como, se ocorre a expressão da proteína do choque térmico (Hsp 70) no fígado e cérebro, como conseqüência da exposição ao estresse por calor, em quatro dias sucessivos (2 a 5 dias de vida), 20 pintos de corte de duas linhagens Pescoço pelado ( $\mathrm{Na} / \mathrm{na}$ ) e Hubbard-Petersen, foram ou não estressados à temperatura de $36-37^{\circ} \mathrm{C}$, durante cinco horas. Foram colhidas amostras de fígado e cérebro, ao final do período de estresse, e analisadas por Western Blotting, para determinação dos níveis de Hsp 70. Os resultados não mostraram associação entre perda de peso corporal e as demais características. Aumentos da variação de temperatura cloacal apresentaram aumentos nos níveis de Hsp70 no tecido hepático e cerebral. Concluiu-se que a concentração de Hsp70 no cérebro, apresentou-se correlacionada com a variação de temperatura cloacal, quando se usou o condicionamento ao calor em pintos de corte, embora não se possa afirmar que seria indicativo de termotolerância de aves.
\end{abstract}

Palavras-chave: Hsp70 hepática e cerebral, estresse por calor, temperatura cloacal, frangos de corte

\section{Physiologic Response Associated to Thermotolerance in Broiler Chickens of Two Strains for Exposure to High Temperature}

\begin{abstract}
This study was run to evaluate the physiological response and expression of hepatic and brain Hsp70, of $20 \mathrm{Naked}$ neck ( $\mathrm{Na} / \mathrm{na}$ ) and Hubbard-Petersen broiler chicks ( 2 to 5 days of age), exposed or not to $36-37^{\circ} \mathrm{C}$. The trial was conducted during four days and the birds were exposed to high temperature during 5 hours. Liver and brain tissue samples were collected at the end of the stress period and analyzed for Western Blotting for determination of Hsp70 level. No association between body weight loss and the other variables were observed. Increasing the variation in cloacal temperature resulted in an increase in Hsp 70 hepatic and brain tissue levels. Results indicate that brain Hsp70 levels were correlated with variation in cloacal temperature, concerning the chicks subjected to heat stress, but it can not be stated that it is an indicative of thermotolerance by the birds.
\end{abstract}

Key Words: broiler chicks, cloacal temperature, heat stress, liver and brain Hsp70 levels

\section{Introdução}

Os frangos de corte adultos são sensíveis a altas temperaturas, tendo seu desempenho prejudicado. Segundo Hurwitz et al. (1980), o desenvolvimento máximo é alcançado em temperaturas entre $18 \mathrm{e}$ $20^{\circ} \mathrm{C}$, ocorrendo elevadas mortalidades, quando a temperatura ambiente excede $38^{\circ} \mathrm{C}$. Fabricio (1994) relatou que o estresse por calor é responsável por grandes perdas no rendimento dos lotes de frangos, ocorrendo aumento de mortalidade e conversão alimentar, além de diminuição do peso corporal, especialmente quando as condições estressantes ocorrem na fase final, próximo ao abate. A termotolerância pode ser melhorada pela aclimatação com exposição dos pintos recém-nascidos (até 5 dias de idade) ao estresse por calor não letal (Arjona et al., 1988).

Hamdy et al. (1991) observaram que a exposição de pintos de corte a temperaturas entre $35-39^{\circ} \mathrm{C}$ nas primeiras 48 horas após a eclosão proporcionou a essas aves na fase adulta, quando expostas a regimes de temperaturas de 35 e $39^{\circ} \mathrm{C}$, temperaturas corporais inferiores às aves do grupo controle $\left(32^{\circ} \mathrm{C}\right)$. Teeter et al. (1992) também encontraram que aves aclimatadas ao calor apresentavam maiores temperaturas cloacais à termoneutralidade e menores temperaturas cloacais quando colocadas em ambientes de $35^{\circ} \mathrm{C}$. Igualmente, aves com o gene $\mathrm{Na}$, o qual confere aos animais redução no empenamento em até $30 \%$, podem apresentar maior perda de calor

1 Doutor pelo Programa de Pós-Graduação em Biotecnologia/UFPel. E.mail: dionello@ufpel.tche.br

2 Departamento de Morfologia e Fisiologia Animal. E.mail: macari@fcav.unesp.br

${ }^{3}$ Departamento de Tecnologia, Faculdade de Ciências Agrárias e Veterinárias, Universidade Estadual Paulista-UNESP, Jaboticabal, SP CEP: 14870-000, Brasil. E.mail: jesus@fcav.unesp.br

${ }^{4}$ Departamento de Zootecnia, Faculdade de Agronomia Eliseu Maciel, Universidade Federal de Pelotas-UFPel, Pelotas, RS. CEP: $96100-000$, Brasil.

E.mail: frutz@ufpel.tche.br

${ }^{5}$ Departamento de Melhoramento e Nutrição Animal, FMVZ-UNESP, Botucatu, SP 
(Cahaner et al., 1993), sendo responsável pelo melhor comportamento dessas aves em condições de estresse pelo calor.

Alguns autores (Lazslo, 1988; Parsell \& Lindquist, 1995) correlacionaram a aquisição de tolerância ao calor com o aumento da síntese da proteína do choque térmico (Hsp70). Evidências de que a proteína Hsp70 está envolvida com a termotolerância foram mostradas em estudos realizados por diversos autores entre eles Arjona et al. (1990), Wang \& Edens (1990) e Miller \& Qureshi (1992). Com relação à duração do estresse por calor Morimoto \& Fodor (1984) observaram que pequenos aumentos de temperatura $\left(2\right.$ a $3{ }^{\circ} \mathrm{C}$ ) foram suficientes para induzir a síntese de Hsp70, quando trabalharam com linfócitos de frangos de corte. Craig \& Gross (1991) sugeriram ainda que as Hsps funcionam como um termômetro celular, sendo induzida sua síntese com o aumento da temperatura. Angelidis et al. (1991), trabalhando com células CV-1 de macacos transfectadas com gene de Hsp70 humanos, verificaram que os clones que foram selecionados pela sua maior síntese de Hsp70 constitutiva, foram os mais resistentes ao calor. Os autores concluíram que a expressão de Hsp70 está relacionada com o mecanismo de proteção celular e pode ser responsável pela termotolerância. Posteriormente, Gabriel (1996), trabalhando com frangos de corte em idades de 1, 21 e 42 dias, relatou que o aumento na temperatura interna de frangos de corte adultos submetidos a um estresse por calor agudo deve atingir determinado limiar para que ocorra aumento nos níveis citoplasmáticos de Hsp70. Dessa maneira, o momento de atingir essa temperatura interna funcionaria como gatilho para síntese induzida desta proteína.

O objetivo do presente trabalho foi avaliar o comportamento fisiológico das aves, bem como, verificar se a expressão da proteína Hsp70 no fígado e cérebro de pintos de corte, de duas linhagens, até 5 dias de idade, ocorre em decorrência de sua exposição ao estresse por calor agudo.

\section{Material e Métodos}

As aves foram criadas no Biotério do Departamento de Tecnologia da Faculdade de Ciências Agrárias e Veterinárias da UNESP - Campus Jaboticabal.

$\mathrm{O}$ estresse, as coletas e análises foram realizados, respectivamente, nos Departamentos de Morfologia e Fisiologia Animal e Tecnologia da UNESP/Jaboticabal.

\section{Estresse por calor}

Oitenta pintos de duas linhagens Pescoço pelado (Na/na) e Hubbard-Petersen, entre 2 e 5 dias de idade, foram estressados ou não a $36-37^{\circ} \mathrm{C}$ durante 5 horas. A temperatura cloacal foi avaliada no início e final do período experimental, por meio de uma sonda termistor introduzida $5 \mathrm{~cm}$ na cloaca e acoplada a um teletermômetro (Yellow Spring Instruments Co.,Ohio, USA). Todos os pintos foram pesados no início e final do estresse por calor, durante o qual foram mantidos sem água e ração. Cinco pintos de cada linhagem e ambiente (total de vinte pintos) foram sacrificados ao final do estresse por calor, em cada dia, e foram colhidas amostras de fígado e cérebro.

As características foram analisadas pelo Estat Sistema para Análises Estatísticas do Departamento de Ciências Exatas da FCAV-UNESP. Para as variáveis temperatura cloacal e peso corporal, antes e depois do estresse, e variação de temperatura corporal e perda de peso corporal, foi realizada análise de variância utilizando-se um arranjo fatorial $2 \times 2 \times 4$, para os efeitos de linhagens (Pescoço pelado e Hubbard-Petersen), ambientes (não estressados e estressados) e idades (2, 3, 4 e 5 dias). O seguinte modelo matemático foi usado:

$$
Y_{i j k l}=\mu+1_{i}+s_{j}+d_{k}+1 s_{i j}+1 d_{i k}+s d_{j k}+
$$

em que: $Y_{i j k l}=$ observação 1 , pertencente à linhagem $\mathrm{i}$, do ambiente $\mathrm{j}$, no dia $\mathrm{k} ; \mathrm{l}_{\mathrm{i}}=$ efeito da linhagem $\mathrm{i}$, $(\mathrm{i}=1,2) ; \mathrm{s}_{\mathrm{j}}=$ efeito do ambiente $\mathrm{j},(\mathrm{j}=1,2) ; \mathrm{d}_{\mathrm{k}}=$ efeito da idade em dias $\mathrm{k},(\mathrm{k}=2,3,4$ e 5$) ; \mathrm{s}_{\mathrm{ij}}=$ efeito da interação linhagem $\mathrm{x}$ ambiente; $1 \mathrm{~d}_{\mathrm{ik}}=$ efeito $\mathrm{da}$ interação linhagem x idade em dias; $\mathrm{sd}_{\mathrm{jk}}=$ efeito da interação ambiente $\mathrm{x}$ idade em dias; $\operatorname{lsd}_{\mathrm{ijk}}=$ efeito da interação linhagem x ambiente $\mathrm{x}$ idade em dias; $\mathrm{e}_{\mathrm{ijkl}}=$ erro experimental, associado a cada observação.

Para a variável idade em dias, como fator quantitativo, foi calculada a regressão, realizando-se também a análise de correlação entre as características variação de temperatura cloacal e perda de peso corporal e os níveis de HSP70 no fígado e cérebro, respectivamente, utilizando-se o programa SAS (1992).

\section{Análise de Western Blotting}

Meio grama de fígado ou cérebro foram homogeneizados, em $5 \mathrm{~mL}$ de tampão de lise $(20 \mathrm{mM}$ tris, $\mathrm{pH} 7,5 ; 0,75 \mathrm{M}$ cloreto de sódio; $2 \mathrm{mM}$ 2-bmercaptoetanol) com um ultra turrax e centrifugado a $30.000 x g$ por 30 minutos a $4^{\circ} \mathrm{C}$. A concentração de proteína do sobrenadante foi quantificada pelo méto- 
do de Hartree (1972) usando soro albumina bovina como padrão. Trinta microgramas de proteína total foram aplicados e separados em gel de 9\% de poliacrilamida contendo SDS (Laemmli, 1970) usando o sistema da Bio-Rad (Mini Protean II). Os géis foram corados com "Coomassie blue" (Morrissey, 1981) e transferidos para membranas de nitrocelulose segundo procedimento proposto por Towbin et al. (1979). As membranas foram coradas com Ponceau S $(0,5 \%$ em ácido acético $1 \%)$ para verificação da eficiência de transferência. Após a remoção do Ponceau $\mathrm{S}$ por lavagem em água deionizada, as membranas foram incubadas em $10 \mathrm{~mL}$ de TBS-leite em pó gelado (10 mM tris, $\mathrm{pH} 8,0 ; 150 \mathrm{mM}$ cloreto de sódio; $5 \%$ leite em pó desnatado e $0,02 \%$ Tween-20) por 1 hora para bloquear sítios de ligação não específicos. As membranas foram então incubadas com 10 $\mathrm{mL}$ de TBS-leite em pó gelado contendo anticorpo monoclonal anti Hsp70 (Sigma H-5147) na diluição de 1:1000. Após a incubação as membranas foram lavadas 4 vezes ( cinco minutos cada) com $10 \mathrm{~mL}$ de TBST gelado (10mM tris, $\mathrm{pH} 8,0 ; 150 \mathrm{mM}$ cloreto de sódio e $0,05 \%$ Tween-20) e 1 vez (10 minutos) com TBS gelado. Adicionou-se o anticorpo secundário anti IgG de camundongo conjugado à fosfatase alcalina (Sigma A-5153) por 1 hora. Após lavagens com TBST e TBS gelada, foi desenvolvida a reação de cor, utilizando os substratos cromogênicos $(0,3 \mathrm{mg} / \mathrm{mL}$ NBT (nitrobluetetrazolium) e $0,15 \mathrm{mg} / \mathrm{mL}$ BCIP (5-bromo-4-cloro-3-indolil fosfato)) em tampão contendo $100 \mathrm{mM}$ tris, $\mathrm{pH} \mathrm{9,5,100} \mathrm{mM}$ cloreto de sódio e $5 \mathrm{mM}$ cloreto de magnésio.

\section{Quantificação das amostras de proteína total}

Os sinais obtidos para análise de Western Blotting foram quantificados por meio de um densitômetro (Model CS-9000, Shimadzu, Japan) e foi determinada a relação de área entre a área de cada amostra e a área da amostra de referência. Utilizando uma curva padrão elaborada para a relação de área entre áreas de diferentes concentrações da proteína Hsp70 em $\mathrm{ng} / \mathrm{mL}$ e a amostra de referência, foi possível obter-se a quantidade de Hsp70 em cada amostra individual de fígado ou cérebro em ng de Hsp70 por mg de proteína total.

\section{Resultados e Discussão}

Os valores de temperaturas cloacais e pesos corporais obtidos, antes e depois do estresse, bem como a variação de temperatura cloacal e perda de peso corporal, estão apresentados nas Tabelas 1 e 2 .

A temperatura cloacal após o estresse por calor nos pintos das duas linhagens, mostrou diferença significativa $(\mathrm{P}<0,05)$ nas diferentes idades, excesso para o quinto dia de idade, enquanto para variação de temperatura cloacal não se encontrou diferença significativa $(\mathrm{P}>0,05)$, dentro de cada idade. Para o efeito de ambientes, os pintos não estressados apresentaram a variação de temperatura cloacal, de acordo com o aumento da idade, variando de forma quadrática $\left(\hat{\mathrm{Y}}=-0,1 \mathrm{x}^{2}+0,372 \mathrm{x}+0,25 ; \mathrm{R}^{2}=30,45\right)$, enquanto para os estressados houve aumento linear da mesma característica $\left(\hat{\mathrm{Y}}=0,218 \mathrm{x}+0,68 ; \mathrm{R}^{2}=97,35\right)$. As diferenças foram significativas $(\mathrm{P}<0,05)$ dentro de cada dia de estresse por calor, exceto para o segundo dia.

As aves de Pescoço pelado apresentaram valores menores de variação de temperatura cloacal em função da idade do que os pintos Hubbard-Petersen, sugerindo que o gene do Pescoço pelado possa estar relacionado com a tolerância ao calor, à semelhança de resultados obtidos por Bordas \& Merat (1984) em poedeiras, Gabriel et al. (1995) em frangos Pescoço pelado e Mazzi (1998), ao compararem os genótipos $\mathrm{Na} / \mathrm{Na}, \mathrm{Na} / \mathrm{na}$ e na/na. Igualmente, os pintos Pescoço pelado podem ter apresentado menor incremento de temperatura cloacal pois produzem menor calor em função da maior perda de calor sensível, o que vem de encontro aos resultados obtidos por Yahav et al. (1998), que indicaram a habilidade dessas aves em manter sua temperatura corporal em altas temperaturas ambientais. Por outro lado, para os pintos Hubbard-Petersen os resultados poderiam significar que essas aves necessitam de um maior tempo para maturação do seu sistema termoregulador.

Os resultados para os pintos estressados para as temperaturas cloacais, depois do estresse térmico, dentro de cada dia de idade, assemelham-se aos obtidos por Givisiez (1997) e Mazzi (1998).

Não houve efeito significativo $(\mathrm{P}>0,05)$ entre linhagens para a perda de peso corporal observada durante o estresse. Entre os ambientes, os pintos estressados apresentaram maior perda de peso corporal $(-6,07 \%>-3,42 \%)$, sendo esta diferença significativa $(\mathrm{P}<0,05)$.

Os pesos corporais observados após o estresse não foram diferentes $(\mathrm{P}>0,05)$ entre os indivíduos estressados ou não, dentro de cada dia de idade. Este 
Tabela 1 - Valores de temperaturas cloacais $\left({ }^{\circ} \mathrm{C}\right)$ dos pintos de corte, antes e depois do estresse, por linhagens, ambientes e idades e respectivos coeficientes de determinação

Table 1 - Cloacal temperature $\left({ }^{\circ} \mathrm{C}\right)$ for broilers chickens, before and after heat stress, for strain, environments and ages, and coefficients of determination

\begin{tabular}{|c|c|c|c|c|c|c|}
\hline \multirow[b]{3}{*}{ Idade (dias) } & \multicolumn{6}{|c|}{$\begin{array}{l}\text { Linhagens } \\
\text { Strain }\end{array}$} \\
\hline & \multicolumn{3}{|c|}{$\begin{array}{l}\text { Pescoço pelado } \\
\text { Naked neck }\end{array}$} & \multicolumn{3}{|c|}{ Hubbard-Pettersen } \\
\hline & A & $\mathrm{D}$ & $\Delta \mathrm{TC}$ & $\mathrm{A}$ & $\mathrm{D}$ & $\Delta \mathrm{TC}$ \\
\hline \multicolumn{7}{|l|}{ Age (days) } \\
\hline 2 & $39,81 \mathrm{a}$ & $40,45 \mathrm{a}$ & $0,64 a$ & $39,79 a$ & $40,67 b$ & $0,88 \mathrm{a}$ \\
\hline 3 & $40,82 \mathrm{a}$ & $41,35 \mathrm{a}$ & $0,53 a$ & $40,05 b$ & $40,93 b$ & $0,88 \mathrm{a}$ \\
\hline 4 & $40,79 a$ & $41,56 a$ & $0,77 \mathrm{a}$ & $39,88 b$ & $41,21 \mathrm{~b}$ & $1,33 \mathrm{a}$ \\
\hline 5 & $41,06 \mathrm{a}$ & $41,57 \mathrm{a}$ & $0,51 \mathrm{a}$ & $40,33 b$ & $41,41 \mathrm{a}$ & $1,08 \mathrm{a}$ \\
\hline \multirow[t]{3}{*}{$\mathrm{R}^{2}$} & 90,23 & 98,58 & 15,74 & 67,43 & 99,84 & 51,69 \\
\hline & \multicolumn{6}{|c|}{$\begin{array}{c}\text { Ambientes } \\
\text { Environments }\end{array}$} \\
\hline & \multicolumn{3}{|c|}{$\begin{array}{c}\text { Não estressado } \\
\text { Control }\end{array}$} & \multicolumn{3}{|c|}{$\begin{array}{c}\text { Estressado } \\
\text { Stressed }\end{array}$} \\
\hline \multicolumn{7}{|l|}{ Age (days) } \\
\hline 2 & $39,74 a$ & $40,38 \mathrm{a}$ & $0,64 a$ & $39,86 a$ & $40,74 b$ & $0,88 \mathrm{a}$ \\
\hline 3 & $40,36 \mathrm{a}$ & $40,60 \mathrm{a}$ & $0,24 b$ & $40,51 \mathrm{a}$ & $41,68 b$ & $1,17 \mathrm{a}$ \\
\hline 4 & $40,16 \mathrm{a}$ & $40,98 \mathrm{a}$ & $0,82 b$ & $40,51 b$ & $41,79 b$ & $1,28 \mathrm{a}$ \\
\hline 5 & $40,90 \mathrm{a}$ & $40,92 \mathrm{a}$ & $0,02 b$ & $40,49 b$ & $42,06 b$ & $1,57 \mathrm{a}$ \\
\hline $\mathrm{R}^{2}$ & 77,76 & 92,42 & 30,45 & 93,61 & 95,05 & 97,35 \\
\hline
\end{tabular}

A - antes do estresse e D - depois do estresse ( $A$ - before heat stress and D - after heat stress).

Para a mesma variável na mesma linha médias seguidas de letras minúsculas diferentes, diferem pelo teste Tukey $(P<0,05)$.

Means, in the lines, followed by different small letters are significantly different $(P<.05)$.

Tabela 2 - Pesos corporais ( $\mathrm{g}$ ) dos pintos de corte, antes e depois do estresse por linhagens, ambientes e idades e respectivos coeficientes de determinação

Table 2 - Body weight $(\mathrm{g})$ for broilers chickens, before and after heat stress, for strain, environment and ages and coefficients of determination

\begin{tabular}{|c|c|c|c|c|c|c|}
\hline \multirow[b]{3}{*}{$\begin{array}{l}\text { Idade (dias) } \\
\text { Age (days) }\end{array}$} & \multicolumn{6}{|c|}{$\begin{array}{l}\text { Linhagens } \\
\text { Strain }\end{array}$} \\
\hline & \multicolumn{3}{|c|}{$\begin{array}{c}\text { Pescoço pelado } \\
\text { Naked neck }\end{array}$} & \multicolumn{3}{|c|}{ Hubbard-Pettersen } \\
\hline & A & $\mathrm{D}$ & $\Delta \mathrm{PC}(\%)$ & $\mathrm{A}$ & $\mathrm{D}$ & $\Delta \mathrm{PC}(\%)$ \\
\hline 2 & $47,13 a$ & $45,64 \mathrm{a}$ & $3,10 \mathrm{a}$ & $55,60 \mathrm{~b}$ & $53,40 \mathrm{~b}$ & $3,95 \mathrm{a}$ \\
\hline 3 & $57,70 \mathrm{a}$ & $54,96 \mathrm{a}$ & $4,75 a$ & $64,27 b$ & $61,56 b$ & $4,13 \mathrm{a}$ \\
\hline 4 & $66,69 \mathrm{a}$ & $63,12 \mathrm{a}$ & $5,28 \mathrm{a}$ & $82,64 b$ & $77,89 \mathrm{~b}$ & $5,73 a$ \\
\hline 5 & $78,94 \mathrm{a}$ & $75,04 \mathrm{a}$ & $4,96 \mathrm{a}$ & $100,88 b$ & $94,80 \mathrm{~b}$ & $6,05 a$ \\
\hline \multirow[t]{3}{*}{$\mathrm{R}^{2}$} & 99,79 & 99,74 & 99,87 & 83,13 & 99,71 & 89,56 \\
\hline & \multicolumn{6}{|c|}{$\begin{array}{c}\text { Ambientes } \\
\text { Environments }\end{array}$} \\
\hline & \multicolumn{3}{|c|}{$\begin{array}{c}\text { Não estressado } \\
\text { Control }\end{array}$} & \multicolumn{3}{|c|}{$\begin{array}{c}\text { Estressado } \\
\text { Stressed }\end{array}$} \\
\hline $\begin{array}{l}\text { Idade(dias) } \\
\text { Age (days) }\end{array}$ & A & $\mathrm{D}$ & $\Delta \mathrm{PC}(\%)$ & A & $\mathrm{D}$ & $\Delta \mathrm{PC}(\%)$ \\
\hline 2 & $50,36 \mathrm{a}$ & $49,26 \mathrm{a}$ & $2,14 a$ & $52,37 \mathrm{a}$ & $49,78 \mathrm{a}$ & $4,91 \mathrm{a}$ \\
\hline 3 & $60,33 a$ & $58,32 \mathrm{a}$ & $3,33 a$ & $61,64 a$ & $58,20 \mathrm{a}$ & $5,56 a$ \\
\hline 4 & $74,73 a$ & $71,56 \mathrm{a}$ & $4,25 \mathrm{a}$ & $74,60 \mathrm{a}$ & $69,45 \mathrm{a}$ & $6,77 \mathrm{a}$ \\
\hline 5 & $92,39 a$ & $88,63 a$ & $3,97 \mathrm{a}$ & $87,43 a$ & $81,21 \mathrm{a}$ & $7,03 \mathrm{a}$ \\
\hline $\mathrm{R}^{2}$ & 99,99 & 1,00 & 98,36 & 99,90 & 99,95 & 96,22 \\
\hline
\end{tabular}

A - antes do estresse e D - depois do estresse ( $A$ - before heat stress and $D$ - after heat stress).

Para a mesma variável na mesma linha, médias seguidas de letras minúsculas diferentes diferem pelo teste de $T u k e y(P<0,05)$.

Means, in the lines, followed by different small letters are significantly different $(P<.05)$. 
conjunto de informações quanto aos pesos corporais não recomendam esta característica como bom indicativo do comportamento fisiológico das aves. Resultados obtidos por Yahav \& Hurwitz (1996), em pintos de corte, mostraram menores pesos corporais após o estresse, especialmente para as aves que durante o período de condicionamento, receberam temperaturas de $36 \pm 1^{\circ} \mathrm{C}$ e $70-80 \%$ UR.

Para ambas as linhagens e ambientes houve um efeito quadrático, para a idade até o quinto dia, apresentando-se os pontos de perda máxima próximos ao final do período observado. De modo geral as aves apresentaram um aumento na perda de peso corporal com o aumento da idade, podendo ser uma maneira de refletir menor tolerância ao calor.

As correlações entre as variações de temperatura cloacal e de peso corporal e as concentrações de hsp70 hepática e cerebral estão apresentadas na Tabela 3.

Apenas a correlação entre variação de temperatura cloacal e concentração de HSP70 no cérebro foi significativa $(\mathrm{P}<0,01)$.

Foram encontradas correlações baixas e negativas, entre a variação de peso corporal e as demais características mostrando pouca associação entre estas variáveis. Esses resultados são indicativos de que, especialmente, os pesos corporais não seriam recomendáveis para serem utilizados como indicativo da tolerância ao calor das aves.

Correlações positivas, embora baixas e não significativas, foram encontradas entre concentração de HSP70 no cérebro e variação de temperatura cloacal $(0,15)$ e concentração de HSP70 no cérebro $(0,16)$, respectivamente. Resultados obtidos por Yahav et al. (1997), em frangos de corte aos 42 dias de idade, mostraram correlação positiva entre a indução da
Hsp70 em tecidos, como coração e pulmões com a temperatura corporal. Igualmente em aves com a idade de 42 dias, Givisiez (1997) encontrou correlação positiva e significativa de 0,39 entre tempo decorrido para aumento da temperatura cloacal em $3^{\circ} \mathrm{C}$ e a concentração de Hsp70 no tecido hepático e Mazzi (1998) encontrou uma relação direta $(0,69)$ entre variação de temperatura cloacal e níveis de Hsp70 em tecido hepático, concluindo os autores que aves com maior variação de temperatura cloacal são as que apresentam maior expressão de Hsp70.

Entretanto, a idade das aves deve ser fator importante a ser observado ao se obterem estas correlações, já que em idades jovens, talvez, elas apresentem um comportamento diverso de quando são adultas (Macari et al., 1994). Sabe-se que a ave necessita de altas temperaturas nos primeiros dias de vida, enquanto estas mesmas altas temperaturas apresentam efeito estressante em frangos de corte. A maior resistência às altas temperaturas pelas aves jovens pode estar relacionada à imaturidade do mecanismo de regulação da temperatura (Dunnington \& Siegel, 1984) e à exigência nessa idade de altas temperaturas para mantença da própria temperatura corporal. Deve-se chamar a atenção quanto a estes aspectos, já que em outros trabalhos, de modo geral, as pesquisas foram realizadas utilizando-se frangos de corte em idades de 21 ou 42 dias.

Os resultados, especialmente para os níveis de Hsp70 no tecido hepático, não comprovam que as proteínas do estresse por calor sejam um fator importante na aquisição de termotolerância em células em cultura e microorganismos conforme mencionado por Lindquist (1986). Concordam principalmente com Yahav et al. (1996), que, ao encontrarem inclusive menor expressão de Hsp70 em indivíduos estressados

Tabela 3 - Estimativas dos coeficientes de correlação entre as características variação de temperatura cloacal $\left({ }^{\circ} \mathrm{C}\right)$ e peso corporal (\%) e níveis de hsp70 hepática e cerebral (ng Hsp70/mg de proteína total)

Table 3 - Correlation between cloacal temperature $\left({ }^{\circ} \mathrm{C}\right)$ and body weight variation (\%) and Hsp70 expression in hepatic and cerebral tissue (ng Hsp70/mg total protein)

Variação de temperatura cloacal(1) Perda de peso corporal (2) Hsp70 de tecido hepático (3) Hsp70 de tecido cerebral (4) Cloacal temperature variation Bodyweight variation Hepatic Hsp70 Cerebral Hsp70

\begin{tabular}{llll}
1 & & & \\
2 & $-0,06$ & $-0,09$ & \\
3 & 0,15 & $-0,01$ & 0,16 \\
4 & $0,32 * *$ & 0 & \\
\hline
\end{tabular}

** $(P<0,01)$. 
do que em não estressados, optaram por concluir que a resposta fisiológica ao estresse por calor em idades jovens, não resulte obrigatoriamente, em menor hipertermia para estas mesmas aves na idade adulta. Igualmente, Yahav et al. (1997) concluíram que não houve resposta da expressão de Hsp70 que representasse uma participação efetiva no mecanismo envolvido com o condicionamento de pintos em idades jovens, especialmente observando-se esta proteína em tecidos como coração, pulmão e linfócitos.

\section{Conclusões}

O comportamento fisiológico das aves frente ao estresse pelo calor pode, principalmente, ser avaliado por meio dos valores de temperatura cloacal obtidos após o estresse térmico, não sendo recomendável a utilização da variação de perda de peso corporal, devido especialmente, a sua fraca associação com as demais características observadas.

Os níveis de Hsp70 no cérebro podem estar correlacionados com a variação de temperatura cloacal, embora não se possa afirmar que seria indicativo de termotolerância das aves.

\section{Agradecimento}

À Fundação de Amparo à Pesquisa do Estado de São Paulo (FAPESP) e à Coordenação de Aperfeiçoamento de Pessoal de Nível Superior (PICD/CAPES), pelo suporte financeiro.

\section{Literatura Citada}

ANGELIDIS, C.E.; LAZARIDIS, I.; PAGOULATOS, G.N. Constitutive expression of heat-shock protein 70 in mammalian confers thermoresistance. European Journal Biochemistries, v.199, n.1, p.670-681.1991.

ARJONA, A.A.; DENBOW, D.M.; WEAVER, Jr., W.O. Effect of heat stress early in life on mortality of broilers exposed to high environmental temperatures just prior to marketing. Poultry Science, v.67, n.2, p.226-231. 1988.

ARJONA, A.A.; DENBOW, D.M.; WEAVER, Jr., W.O. Erythrocyte protein profile of neonatally stressed broilers exposed to a juvenile heat challenge. Poultry Science, v.69, n.1, p.9, 1990. (abstract)

BORDAS, A.; MERAT, P. Effects of naked-neck gene on traits associated with egg laying in dwarf stock at two temperatures. British Poultry Science, v.25, p.195-207, 1984.

CAHANER, A.; DEEB, N.; GUTMAN, M. Effects of the plumage-reducing naked neck $(\mathrm{Na})$ gene on the performance of fast-growing broilers at normal and high ambient temperatures. Poultry Science, v.72, n.5, p.767-775. 1993.

CRAIG, E.A.; GROSS, C.A. Is Hsp70 the cellular thermometer? Tibs, v.16, p.135-140, 1991.
DUNNINGTON, E.A.; SIEGEL, P.B. Thermoregulation in newly hatched chicks. Poultry Science, v.63, n.9, p.1303-1313, 1984.

FABRICIO, J.R. Influência do estresse calórico no rendimento da criação de frangos de corte. In: CONFERÊNCIA APINCO DE CIÊNCIA E TECNOLOGIA AVÍCOLAS, Santos, 1994. Anais... Campinas: FACTA, 1994. p.129-136.

GABRIEL, J.E. Efeitos do nível energético da ração e do estresse térmico na expressão da proteína de choque térmico Hsp70 e nos níveis de seu mRNA no fígado de frangos de corte em diferentes estágios de desenvolvimento. Jaboticabal: Universidade Estadual Paulista, 1996. 84 p. Dissertação (Mestrado em Zootecnia) - Universidade Estadual Paulista, 1996.

GABRIEL, J.E.; GUERREIO, E.N.; SILVA, M.M. et al. Padrão de expressão da proteína de choque térmico de $70 \mathrm{kDa}$ (Hsp70) em aves selecionadas e não selecionadas submetidas a um estresse calórico agudo. In: CONFERÊNCIA APINCO DE CIÊNCIA E TECNOLOGIA AVÍCOLAS, 1995, Curitiba. Anais... Campinas: FACTA, 1995. p.303-304.

GIVISIEZ, P.E.N. Mecanismos hormonais e moleculares da termotolerância em frangos de corte. Jaboticabal: Universidade Estadual Paulista, 1997. 77p. Dissertação (Mestrado em Zootecnia) - Universidade Estadual Paulista, 1997.

HAMDY, A.M.M.; Van DER HEL; HENKEN, A.M. et al. Effect of air humidity during incubation and age after hatch on heat tolerance of neonatal male and female chicks. Poultry Science, v.70, n.7, p.1499-1506, 1991.

HARTREE, E.F. Determination of protein: a modification of the Lowry method that gives a linear photometric response. Analytical Biochemistry, v.48, n.2, p.422-427, 1972.

HURWITZ, S.; WIESELBERG, M.; EISNER, U. et al. The energy requirements and performance of growing chickens and turkeys, as affected by environmental temperature. Poultry Science, v.59, n.9, p.2290-2299, 1980.

LAEMMLI, U.K. Cleavage of structural proteins during the assembly of the head of bacteriophage T4. Nature, v.227, p.680-685, 1970.

LASZLO, A. The relationship of heat-shock proteins, thermotolerance, and protein synthesis. Experimental Cell Research, v.178, p.401-414, 1988.

LINDQUIST, S. The heat-shock responses. Annual Review of Biochemistry, v.55, p.1151-1191, 1986.

MACARI, M.; FURLAN, R.L.; GONZALEZ. Fisiologia aviária aplicada a frangos de corte. Jaboticabal: Universidade Estadual Paulista, 1994. 296p.

MAZZI, C.M. Análise da expressão da proteína de estresse Hsp70 em frangos de corte portadores do gene "naked neck"(pescoço pelado) submetidos a estresse térmico gradativo. Jaboticabal: Universidade Estadual Paulista, 1998. 60p. Dissertação (Mestrado em Agronomia) - Universidade Estadual Paulista, 1998.

MILLER, L.; QURESHI, M.A. Heat-shock protein synthesis and phagocytic function of chicken macrophage following in vitro heat exposure. Veterinary Immunologic Immunopathology, v.30, p.179-191, 1992.

MORIMOTO, R.I.; FODOR, E. Cell-specific expression of heat shock proteins in chicken reticulocytes and lymphocytes. Journal Cellular Biology, v.99, p.1316-1323, 1984.

MORRISSEY, D.H. Silver strain for proteins in polyacrilamide gels: a modified procedure with enhanced uniform sensitivity. Analytical Biochemistry, v.117, p.307-310, 1981.

PARSELL, D.A., LINDQUIST, S. Heat shock proteins and stress tolerance. In: The biology of heat shock proteins and molecular chaperones. Cold Spring Harbor: Cold Spring Harbor Laboratory Press, 1995. p.457-495. 
SAS INSTITUTE. Statistical Analysis System. Users guide. Cary: 1992.

TEETER, R.G.; SMITH, M.O.; WIERNUSK, C.J. Broiler acclimation to heat distress and feed intake. Effects on body temperature in birds exposed to termoneutral and high ambient temperatures. Poultry Science, v.71, n.6, p.1101-1104. 1992.

TOWBIN, H.; STAELIN, T.; GORDON, J. Electrophoretic transfer of proteins from polyacrylamide gels to nitrocellulose sheets: procedure and some applications. Proceedings National Academic Science United State, v.76, n.9, p.43504354. 1979.

WANG, S.; EDENS, F.W. Heat shock proteins induced in blood cells of cockerels. Poultry Science, v.19, n.1, p.196, 1990. (abstract)

YAHAV, S.; HURWITZ, S. Induction of thermotolerance in male broiler chickens by temperature conditioning at an early age. Poultry Science, v.75, n.3, p.402-405, 1996.
YAHAV, S.; LUGER, D.; CAHANER, A. et al. Thermoregulation in naked neck chickens subjected to different ambient temperatures. British Poultry Science, v.39, p.133-138. 1998.

YAHAV, S.; SHAMAY, A.; HABERFELD, A. et al. Induction of thermotolerance in chickens by temperature conditioning: heat shock protein expression. Annals New York Academy of Science, p.628-636, 1996.

YAHAV, S.; SHAMAY, A.; HOREV, G. et al. Effect of acquisition of improved thermotolerance on the induction of heat shock protein in broiler chickens. Poultry Science, v.76, n.10, p.1428-1434, 1997.

Recebido em: 19/09/00

Aceito em: 27/08/01 